\title{
EGstudios Gerenciales
}

Journal of Management and Economics for Iberoamerica

Artículo de investigación

\section{Ética corporativa y precio justo percibido, el binomio estratégico para el consumo responsable}

\author{
Lucirene Rangel-Lyne* \\ Profesora investigadora, Área de Negocios y Humanidades, Universidad del Noreste, Tampico, México \\ Profesora investigadora, Facultad de Comercio y Administración de Tampico, Universidad Autónoma de Tamaulipas, Tampico, México. \\ lucirene.rangel@une.edu.mx
}

José Ignacio Azuela-Flores

Profesor investigador, Facultad de Comercio y Administración de Tampico, Universidad Autónoma de Tamaulipas, Tampico, México.

iazuelaf@docentes.uat.edu.mx

Magda Lizet Ochoa-Hernández

Profesora investigadora, Facultad de Comercio y Administración de Tampico, Universidad Autónoma de Tamaulipas, Tampico, México.

mlochoa@docentes.uat.edu.mx

Mirtha Elba Infante-Aguillón

Profesora y directora de carrera, Área de Negocios y Humanidades, Universidad del Noreste, Tampico, México.

mirtha.infante@une.edu.mx

\section{Resumen}

La responsabilidad corporativa ética eleva la percepción de justicia en los precios de los productos adquiridos. El propósito de este artículo fue confirmar dicha premisa como predictora de la intención de recompra socialmente responsable. Se analizaron las percepciones de 153 consumidores con poder adquisitivo restringido, que declararon comprar productos de empresas con responsabilidad ética. El efecto del precio justo percibido se demostró en una mediación total para explicar las intenciones de recompra socialmente responsables.

Palabras clave: responsabilidad corporativa ética; precio justo; consumidores.

\section{Corporate ethics and perceived fair price, the strategic binomial for responsible consumption}

\section{Abstract}

Ethical corporate responsibility elevates the price fairness perception of the purchased products. This paper aimed to confirm this premise as a predictor of the socially responsible repurchase intention. The perceptions of 153 consumers with restricted purchasing power, who declared to buy products from companies with ethical responsibility, were analyzed. The effect of the perceived fair price was demonstrated in a full mediation to explain socially responsible repurchase intentions.

Keywords: ethical corporate responsibility; fair price; consumers.

\section{Ética corporativa e preço justo percebido, o binômio estratégico para o consumo responsável}

Resumo

A responsabilidade corporativa ética eleva a percepção de justiça nos preços dos produtos adquiridos. 0 objetivo deste artigo foi confirmar essa premissa como um preditor da intenção de recompra socialmente responsável. Analisaram-se as percepções de 153 consumidores com poder aquisitivo restrito, que declararam adquirir produtos de empresas com responsabilidade ética. 0 efeito do preço justo percebido foi demonstrado em mediação completa para explicar as intenções de recompra socialmente responsáveis.

Palavras-chave: responsabilidade corporativa ética; preço justo; consumidores

* Autor para dirigir correspondencia.

Clasificación JEL: M14; M2; C0; D12.

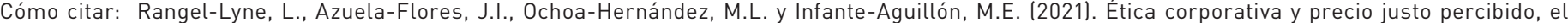
binomio estratégico para el consumo responsable. Estudios Gerenciales, 37(160), 439-447. https://doi.org/10.18046/j.estger.2021.160.4098

DOI: https://doi.org/10.18046/j.estger.2021.160.4098

Recibido: 19-jun-2020

Aceptado: 18-ene-2021

Publicado: 31-ago-2021 


\section{Introducción}

La evidencia empírica previa ha identificado que los esfuerzos éticos corporativos, cuando son percibidos por los consumidores, se convierten en un incentivo hacia la compra, incluso generando una propensión a pagar un poco más por los productos que las empresas ofertan (Amezcua, Briseño, Ríos y Ayala, 2018).

Lo anterior se ve reflejado en la disminución de quejas emitidas a corporaciones responsables, pues el cumplimiento de las obligaciones éticas genera un bien común que se traduce en la mejor valoración de este tipo de empresas (Carvalho, Sen, de Oliveira Mota y de Lima, 2010; Elgammal, El-Kassar y Messarra, 2018; Gómez y Martínez, 2016).

La ética está intrínsecamente asociada con la responsabilidad social corporativa (RSC) y la literatura previa ha propuesto el estudio de la ética como una de sus dimensiones (Carroll, 1979). Puesto que la ética es una dimensión de la RSC, esta se ha denominado responsabilidad corporativa ética (RCE), pues conlleva una responsabilidad inherente a su significado.

Para fines del presente estudio, la RCE se entiende como el apego a la ley y el respeto al derecho de todos los grupos interesados, que da prioridad a tal cumplimiento con honestidad, por encima del desempeño económico (De Los Salmones, Crespo y Del Bosque, 2005; Gómez y Martínez, 2016; Maignan, 2001; Maignan y Ferrell, 2004).

La RCE ha sido un elemento clave para la subsistencia de los entes económicos, pues mediante su adopción se logra la aceptación de la sociedad en la que se desarrollan. Es decir, los individuos de tal sociedad en su rol de consumidores evalúan a dichas corporaciones de manera satisfactoria. Esto sugiere una mayor intensidad en el contexto actual, pues se evidencia una sociedad de consumo más exigente que está legitimando sus percepciones mediante sus consumos (Koellner, 2019), lo que merece especial atención en escenarios en los que prácticas corporativas no éticas hayan sido expuestas (ArellanoGault, 2019).

Pese a lo anterior, existen variables alternas que se evidencian como discriminantes antes de la compra. Las más evidentes se relacionan con el poder adquisitivo, por lo que es necesario considerar variables determinantes de las intenciones de compra, de acuerdo a los ingresos de los consumidores.

El precio es una de las principales variables que, en general, los consumidores refieren como un factor discriminante antes de la compra, pues su sensibilidad aumenta a medida que el ingreso se reduce (MarquinaFeldman y Reficco, 2015). Este se ha percibido como excesivo, justo o bajo. La delgada línea que diferencia una percepción de la otra se debe a motivantes internos y externos. Respecto a los motivantes internos se encuentran factores culturales como el individualismo, el colectivismo, los valores (Díaz, Díaz y González, 2017) y las características de una tipología de consumidor exigente de un comportamiento corporativo responsable (Culiberg y Mihelic, 2016). Respecto a los externos, se identifican, por ejemplo, las exposiciones y estímulos mediáticos corporativos (Sen y Bhattacharya, 2001).

Dichas exposiciones y estímulos se convierten en percepciones que, en complemento de los motivantes internos, generan efectos que explican el consumo (Dopico, Rodríguez y González, 2014). Entonces, con base en lo expuesto, se afirma que la percepción del precio, como uno de los discriminantes principales previos a la compra se ve modificada al percibir una alta RCE. En otras palabras, cuando la RCE es percibida por los consumidores, el precio tiende a percibirse como justo.

La presente investigación tuvo como objetivo analizar el efecto mediador del precio justo percibido (PJP) en la relación entre la RCE y la intención de recompra socialmente responsable (IRSR).

En el artículo se desarrolla una breve discusión acerca de la RCE percibida y el efecto mediador del PJP en el consumo socialmente responsable, luego se presenta una descripción del método, seguida del análisis de los resultados y, por último, de las conclusiones.

\section{Marco conceptual}

El precio es uno de los discriminantes básicos para la realización de cualquier compra. Este se encuentra directamente relacionado con una percepción de valor que se comprende como un juicio comparativo, con respecto al precio de referencia en el mercado (Grewal, Krishnan, Baker y Borin, 1998).

Atendiendo a los diferentes precios de referencia y a la diversidad de valoraciones en la mente de los consumidores, las percepciones de precios dependen del valor atribuido a los productos (Habel, Schons, Alavi y Wieseke, 2015), mientras que investigaciones alternas argumentan que la percepción del precio depende del nivel de comprensión que los consumidores mantengan respecto de su fijación (Jung, Cho y Jin, 2020).

El valor de los productos se incrementa debido a distintas razones. Estudios previos confirman que las dimensiones de la RSC mantienen una incidencia positiva en el valor percibido; es decir, cuando los consumidores perciben que un producto fue elaborado a través de acciones responsables, el valor de tal producto tenderá a incrementarse. Por consiguiente, el esfuerzo (Berry, Seiders y Grewal, 2002) y el sacrificio (Zeithaml, 1988) por la adquisición de ese bien se verán justificados, argumento válido para efectuar la compra.

Una de las dimensiones de la RSC que mayor incidencia mantiene en las evaluaciones positivas de los consumidores es la dimensión ética (Marquina- 
Feldman y Reficco, 2015). Estudios realizados por Peloza, White y Shang (2013) sugieren que los atributos corporativos éticos motivan las preferencias de compra de los consumidores, incluso por encima de las percepciones de beneficio tradicionales, propias del desempeño del producto (Peloza et al., 2013).

El precio que es evaluado a partir de una base corporativa ética tiene altas posibilidades de ser percibido como justo. El precio justo se define como un aspecto cognitivo que es producto de los juicios comparativos del mismo precio y de procedimientos con estándares, referencias o normas (Xia, Monroe y Cox, 2004). Esto se confirma mediante estudios recientes que incluyen valoraciones de precios en el mercado de consumo (Zietsman, Mostert y Svensson, 2019), estudios que incluso argumentan que esta clase de percepciones se intensifican en el contexto actual, debido a una mayor sensibilidad por parte de los consumidores ante la injusticia en los precios (Chark, 2019).

Así pues, la percepción sobre el monto a pagar es favorable, es decir, se considera menor, para los productos con perfil corporativo ético (Ferrell, Harrison, Ferrell y Hair, 2019), con lo cual, crece la aceptación hacia los precios cuando los consumidores conocen las acciones responsables éticas por parte de la compañía (Semuel y Chandra, 2014). Lo anterior ha confirmado a la ética en un nivel determinante para construir una responsabilidad social en el mercado de consumo (Arli y Tjiptono, 2018).

La literatura previa confirma, además, que cuando el precio se respalda por una proyección ética, este se percibe como un precio justo $y$, por tanto, los consumidores demuestran una disposición a pagar, incluso un precio más alto (Habel et al., 2015). Lo anterior se evidencia a través de las percepciones de un mayor beneficio, así como en mejores evaluaciones cuando un incremento en el precio es percibido como justo (Abrantes, Gonçalves y Dias, 2010).

Una mayor percepción de justicia en el precio incentiva no solo a la intención de compra, sino también la generación de lealtad hacia las compañías socialmente responsables (Habel et al., 2015; Marquina-Feldman y Reficco, 2015). Lo anterior se muestra incluso en estudios que contemplan percepciones de beneficio tradicional como la calidad (Habel et al., 2015; Matute-Vallejo, Bravo y Pina, 2011).

El efecto catalizador del precio justo percibido sobre la intención de compra se ha destacado como una variable mediadora que muestra efectos positivos y significativos en el comportamiento de compra (Konuk, 2019), especialmente en el segmento de consumidores con un alto sentido de autorresponsabilidad (Peloza et al., 2013). Este efecto catalizador ha sido confirmado inclusive con mayor fuerza en los consumidores que cuentan con mayores restricciones en su gasto que en consumidores con ingresos altos (Carvalho et al., 2010).
En resumen, cuando los consumidores logran percibir una RCE, estos son capaces de percibir PJP, que actúa como un incentivo hacia la compra socialmente responsable. Estas percepciones logran generar una IRSR en quienes ya consumen productos de las compañías que se desarrollan con una responsabilidad ética (figura 1). A partir de lo discutido, se propone que:

- $H_{1}$ : la RCE percibida tiene efectos positivos en la intención de recompra socialmente responsable.

- $\mathrm{H}_{2}$ : el PJP media la relación entre RCE percibida y la IRSR.

\section{Metodología}

El diseño metodológico propone comprobar las hipótesis expuestas en el apartado anterior. Si la RCE percibida por los consumidores es la generadora de un PJP, esta última percepción propicia las intenciones de IRSR de los consumidores de ingresos restringidos en el contexto mexicano. Lo anterior se muestra en un modelo de ecuaciones estructurales basado en covarianzas (figura 1).

\subsection{Método}

El diseño fue cuantitativo, explicativo y transversal. El modelo propuesto fue analizado mediante un análisis factorial exploratorio (AFE) en SPSS (Statistical Package for the Social Sciences) versión 24, un análisis factorial confirmatorio (AFC) y un modelo de ecuaciones estructurales en el software AMOS (Analysis of Moment Structures), versión 24.

\subsection{Muestra}

Mientras los consumidores de ingresos altos ejercen con mayor libertad sus consumos con base en los gustos y preferencias, los consumidores de ingresos menores consideran mayores restricciones en su gasto al momento de seleccionar sus productos (Carvalho et al., 2010; Higuchi, 2015). Con base en lo anterior, el estudio de este fenómeno cobra relevancia en países que cuentan con consumidores de un poder adquisitivo restringido.

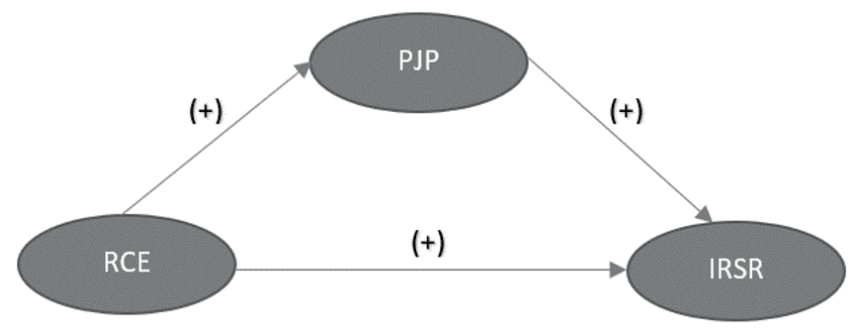

Figura 1. Modelo de Responsabilidad Corporativa Ética (RCE), Precio Justo Percibido (PJP) e Intención de Recompra Socialmente Responsable (IRSR)

Fuente: elaboración propia. 
México es un país clasificado como una economía de ingresos medios superiores, calculados con base en el ingreso nacional bruto ( $G N I$, por sus siglas en inglés) per cápita (The World Bank, 2020).

Debido a que la variable PJP requiere ser medida a partir de las evaluaciones de quienes consumen productos de empresas percibidas como éticas y responsables, la muestra se enfocó en este segmento de consumidores.

La aplicación de las encuestas tuvo lugar principalmente en universidades, centros sociales y comerciales del sur de Tamaulipas, México. Los que consumen productos de compañías socialmente responsables se identificaron mediante una pregunta dicotómica en la encuesta. Es decir, después de que los sujetos hicieran mención de las compañías que perciben como éticas, se cuestionó si habían efectuado consumos en ellas, en el último mes.

Se encuestó a un total de 450 mexicanos. En la selección de la muestra final se consideró principalmente 1) a quienes afirmaron consumir productos de las empresas que perciben como éticas, 2) a quienes declararon estar trabajando, 3) a quienes tuvieron la disposición de mencionar su ingreso y 4 l) a quienes su ingreso salarial no fuera alto. Es necesario precisar que uno de los mayores problemas en los criterios de selección obedeció a una alta omisión de respuesta en la pregunta que cuestionaba el ingreso del consumidor.

La muestra final incluyó a 153 trabajadores mexicanos (ingresos: MXN 4999-9999), que consumen productos de compañías socialmente responsables (tabla 1).

\subsection{Medidas}

Las medidas fueron adaptadas a partir de conceptos y escalas de investigaciones previas. Para medir la RCE se incluyeron indicadores de los trabajos de De Los Salmones et al. (2005), Maignan (2001) y Maignan y Ferrell (2004). La escala de PJP se adecuó desde los estudios de Matute-Vallejo et al. (2011) y Bolton, Warlop y Alba (2003) en Habel et al. (2015).

Para medir la intención de recompra se incluyeron ítems adaptados de las investigaciones de Berens, Van Riel y Van Bruggen (2005); Habel et al. (2015) y Tian, Wang y Yang (2011) (tabla 2).

\subsection{Instrumento}

La encuesta incluyó en primer lugar los datos demográficos, seguidos de la pregunta filtro de consumo responsable, para después continuar con la inclusión de las variables latentes. En primer lugar, se presentó la escala que evalúa la intención de recompra. En segundo lugar, se ubicó la escala de RCE percibida para finalmente concluir con la escala de PJP.
Tabla 1. Descripción de la muestra $(n=153)$

\begin{tabular}{|c|c|}
\hline Variables demográficas & Frecuencia \\
\hline \multicolumn{2}{|l|}{ Sexo } \\
\hline Hombre & 51 \\
\hline Mujer & 102 \\
\hline \multicolumn{2}{|l|}{ Edad } \\
\hline De 55 a 73 años & 8 \\
\hline De 39 a 54 años & 36 \\
\hline De 19 a 38 años & 102 \\
\hline De 15 a 18 años & 7 \\
\hline \multicolumn{2}{|l|}{ Ciudad de residencia } \\
\hline Tampico & 89 \\
\hline Madero & 16 \\
\hline Altamira & 48 \\
\hline \multicolumn{2}{|l|}{ Estado Civil } \\
\hline Soltero & 94 \\
\hline Casado & 44 \\
\hline Otro & 15 \\
\hline \multicolumn{2}{|l|}{ Labora } \\
\hline No & 0 \\
\hline Sí & 153 \\
\hline \multicolumn{2}{|l|}{ Escolaridad } \\
\hline Secundaria & 15 \\
\hline Preparatoria & 76 \\
\hline Universidad & 47 \\
\hline Maestría & 15 \\
\hline Doctorado & 0 \\
\hline \multicolumn{2}{|l|}{ Salario mensual (MXN) } \\
\hline$<\$ 4.999$ & 86 \\
\hline $5.000-9.999$ & 67 \\
\hline $10.000-14.999$ & 0 \\
\hline$>\$ 15.000$ & 0 \\
\hline
\end{tabular}

Fuente: elaboración propia.

La encuesta fue autoadministrada en papel. Los constructos de variables latentes se diseñaron con base en una escala Likert de siete puntos: 1= Totalmente en desacuerdo; $2=$ En desacuerdo; $3=$ Ligeramente en desacuerdo; $4=\mathrm{Ni}$ en acuerdo $\mathrm{ni}$ en desacuerdo; $5=$ Ligeramente de acuerdo; $6=$ De acuerdo y $7=$ Totalmente de acuerdo. Se optó por diseñar el instrumento con base en una escala de siete puntos, en concordancia con la literatura de estudios alternos que manejan percepciones, actitudes e intenciones de compra responsable (Brown y Dacin, 1997; Habel et al., 2015; Maignan, 2001; Martínez y Rodríguez del Bosque, 2013).

Se efectuó una prueba piloto con 20 consumidores. Después de tal prueba se modificaron algunos ítems para una mejor comprensión. Posteriormente, la encuesta fue revisada por expertos.

\section{Análisis de los resultados}

La muestra final de consumidores identificó como empresas éticas a las grandes multinacionales de la industria de bebidas, comercio y restaurantes, que, en efecto, cuentan con el distintivo de empresa socialmente responsable (ESR). 
Tabla 2. Operacionalización de las variables latentes

\begin{tabular}{|c|c|c|c|}
\hline Variable latente & Código & Indicadores & Adecuado de: \\
\hline \multirow{4}{*}{$\begin{array}{l}\text { Intención de } \\
\text { Recompra } \\
\text { Socialmente } \\
\text { Responsable } \\
\text { (Código: IRSR) }\end{array}$} & 1. INT1: & Es muy posible que compre productos de una empresa socialmente responsable & \multirow{4}{*}{$\begin{array}{l}\text { Berens et al. (2005); } \\
\text { Tian, Wang y Yang. } \\
\text { (2011) y Habel et al. } \\
\text { (2015). }\end{array}$} \\
\hline & 2. INT2: & $\begin{array}{l}\text { Estaría dispuesto a pagar un poco más por adquirir productos de una empresa } \\
\text { socialmente responsable }\end{array}$ & \\
\hline & 3. INT3: & Compraré productos de una empresa socialmente responsable & \\
\hline & & Yo creo que una empresa socialmente responsable... & \\
\hline \multirow{3}{*}{$\begin{array}{l}\text { Responsabilidad } \\
\text { Corporativa Ética } \\
\text { (Código: RCET) }\end{array}$} & 4. RSCET1 & $\begin{array}{l}\text { Siempre respeta las normas definidas en la ley cuando desempeña sus } \\
\text { actividades }\end{array}$ & \multirow{3}{*}{$\begin{array}{l}\text { Maignan (2001); } \\
\text { Maignan y Ferrel (204) } \\
\text { y De Los Salmones et } \\
\text { al. (2005). }\end{array}$} \\
\hline & 6. RSCET3 & Se comporta ética y honestamente con sus consumidores & \\
\hline & 7. RSCET4 & Da prioridad a sus principios, por encima de su desempeño económico & \\
\hline $\begin{array}{l}\text { Precio Justo } \\
\text { Percibido } \\
\text { (Código: PJP) }\end{array}$ & 8. PJ1: & $\begin{array}{l}\text { Considero que el precio que pago por productos de empresas socialmente } \\
\text { responsables es justo. }\end{array}$ & $\begin{array}{l}\text { Matute et al. (2010), } \\
\text { Bolton et al. (2003) en } \\
\text { Habel et al. (2015). }\end{array}$ \\
\hline
\end{tabular}

Fuente: elaboración propia con base en escalas de estudios previos.

Para realizar el AFE, se incluyeron las escalas de PJP, IRSR y la responsabilidad corporativa ética (RCET). La medida de Kaiser-Mayer-Olkin de adecuación de muestreo fue de 0,844 y la prueba de esfericidad de Barlett resultaron significativas $(p=$ $0,000)$, lo que hace viable el análisis.

El método de extracción de factores fue el de máxima verosimilitud, basado en el autovalor. La rotación aplicada fue promax. La varianza explicada obtenida fue de $65,24 \%$. El primer factor $(42,70 \%)$ agrupó los cuatro ítems de la escala de RCE, el segundo factor $(12,20 \%)$ logró incluir los indicadores del PJP, mientras que el tercer factor $(10,34 \%)$ agrupó los reactivos de la IRSR. Los valores de las cargas factoriales obtenidas fueron cercanos a la unidad. Ninguno de los indicadores se agrupó en dos factores, con lo que la conformación factorial fue clara (tabla 3).

Tabla 3. Análisis Factorial Exploratorio

\begin{tabular}{lccc}
\hline & \multicolumn{3}{c}{ Factor } \\
& 1 & 2 & 3 \\
\hline RSCET2 & 0,868 & & \\
RSCET1 & 0,842 & & \\
RSCET3 & 0,828 & & \\
RSCET4 & 0,634 & & \\
PJ2 & & 0,933 & \\
PJ1 & & 0,853 & \\
PJ3 & & 0,734 & 0,767 \\
INT3 & & & 0,706 \\
INT1 & & & 0,658 \\
INT2 & & & \\
\hline
\end{tabular}

Método de extracción: máxima verosimilitud. Método de rotación: Promax con normalización Kaiser. La rotación ha convergido en 5 iteraciones.

Fuente: elaboración propia con base en SPSS versión 24.
Al realizar el AFC en AMOS, las cargas de cada indicador fueron en todos los casos mayores a 0,707 , con excepción de un indicador de la variable de RCET (RSCET4) y dos indicadores pertenecientes a la escala de IRSR (INT1; INT2). No obstante, con base en el número mínimo de ítems sugerido para la conformación de variables latentes y la explicación que aportan dichos ítems de acuerdo con la literatura revisada, se decidió no efectuar eliminaciones (figura 2).

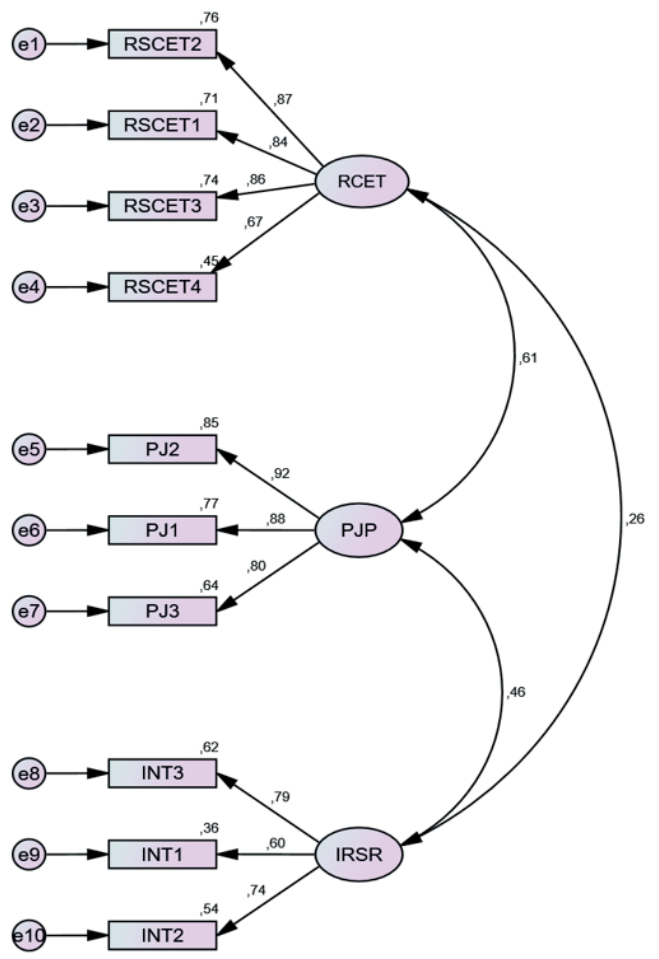

Figura 2. Modelo de medida

Fuente: AMOS versión 24. 
Para confirmar la fiabilidad de las escalas se realizó el estadístico de fiabilidad alfa de Cronbach y se obtuvo $a=0,748$ para la variable de IRSR; $a=$ 0,881 para la RCET y a $=0,898$ para el PJP.

La validez compuesta (CR) obtenida fue mayor a 0,7 en todas las escalas. Al probar la validez convergente mediante la varianza media extraída (AVE, por sus siglas en inglés), esta fue mayor a 0,5 en todos los casos, lo que las hace aceptables (Chin, 1998). Finalmente, para evaluar la validez discriminante de las variables latentes se siguió el criterio Fornell-Larcker, que sugiere comparar la raíz cuadrada de la AVE con las correlaciones entre constructos; este criterio se satisface siempre que la raíz cuadrada del AVE sea mayor que las correlaciones entre constructos. Los resultados muestran que la raíz cuadrada de la AVE en todos los casos es superior a las correlaciones entre constructos (tabla 4).

Al realizar el modelo estructural, se revisaron los indicadores de ajuste del modelo. El chi cuadrado normalizado por los grados de libertad se obtuvo dentro del parámetro de 1 a 3; el error de la raíz media de aproximación (RMSEA) obtuvo valores menores a 0,06 , que confirman un buen ajuste del modelo (Baumgartner y Homburg, 1996); y el índice de ajuste comparativo (CFI) estuvo ligeramente por encima del 0,95 , por lo que se aconseja la aceptación del modelo propuesto (tabla 5).

Tabla 4. Validez de las escalas de variables latentes

\begin{tabular}{llllllll}
\hline & CR & AVE & MSV & MaxR(H) & PJP & IRSR & RCET \\
\hline PJP & 0,900 & 0,752 & 0,368 & 0,914 & 0,867 & & \\
IRSR & 0,754 & 0,509 & 0,214 & 0,773 & $0,462^{* * *}$ & 0,713 & \\
RCET & 0,887 & 0,665 & 0,368 & 0,902 & 0,607 & 0,258 & 0,815 \\
\hline
\end{tabular}

$* * * p<0,001$

Fuente: AMOS versión 24.

Tabla 5. Índices de ajuste del modelo estructural

\begin{tabular}{lcc}
\hline Medida & Estimación & Referencia \\
\hline CMIN/DF & 1,023 & Entre 1 y 3 \\
CFI & 0,999 & $>0,95$ \\
RMSEA & 0,012 & $<0,06$ \\
PClose & 0,865 & $>0,05$ \\
\hline
\end{tabular}

Fuente: AMOS versión 24.

El efecto directo estandarizado de RCET sobre IRSR es $-0,04$. El efecto indirecto estandarizado de RCET sobre IRSR se define como el producto de dos efectos directos estandarizados: el efecto directo estandarizado de RCET sobre PJP $(0,61)$ y el efecto directo estandarizado de PJP sobre IRSR $(0,48)$. El producto de estos dos efectos directos estandarizados es igual a 0,29; el efecto indirecto estandarizado total de la mediación.

De manera complementaria y únicamente para evidenciar el efecto catalizador del PJP, se reprodujo la relación directa de la RCET sobre la IRSR. En este modelo se confirma que la relación entre la RCET y la IRSR es positiva y significativa, al no contar con el PJP como variable mediadora. Sin embargo, los efectos entre ambas variables presentan una $R^{2}$ estandarizada de 0,07, lo que refiere un efecto débil (figura 4).

En el modelo de mediación (figura 3) se demuestra que la relación entre RCET y PJP es positiva y significativa $\left(B=0,607 ; p=0,000 ; R^{2}=0,37\right)$; se confirma como un binomio que explica a la IRSR $(B=$ 0,$484 ; p=0,000 ; R^{2}=0,21$ ) (tabla 6). No obstante, la relación directa entre RCET e IRSR no fue significativa $(B=-0,036 ; p>0,05)$. Esto se debe a que el PJP absorbió y catalizó el efecto emitido por la RCET sobre la IRSR, tal como se presenta en la comparación de los modelos reproducidos (figura 3 y 4 ).

Finalmente, el efecto mediador del PJP se demuestra con el cambio en la $\mathrm{R}^{2}$ estandarizada de apenas 0,07 en el modelo que excluye tal variable; en contraste con una $R^{2}$ estandarizada de 0,21 al incluir al PJP como mediador para explicar la IRSR. Considerando que, para estudios relacionados con el comportamiento del consumidor, una $\mathrm{R}^{2}$ de 0,20 es considerada aceptable (Hair, Hult, Ringle y Sarstedt, 2017), el rol del PJP se confirma como una variable imprescindible para las corporaciones.

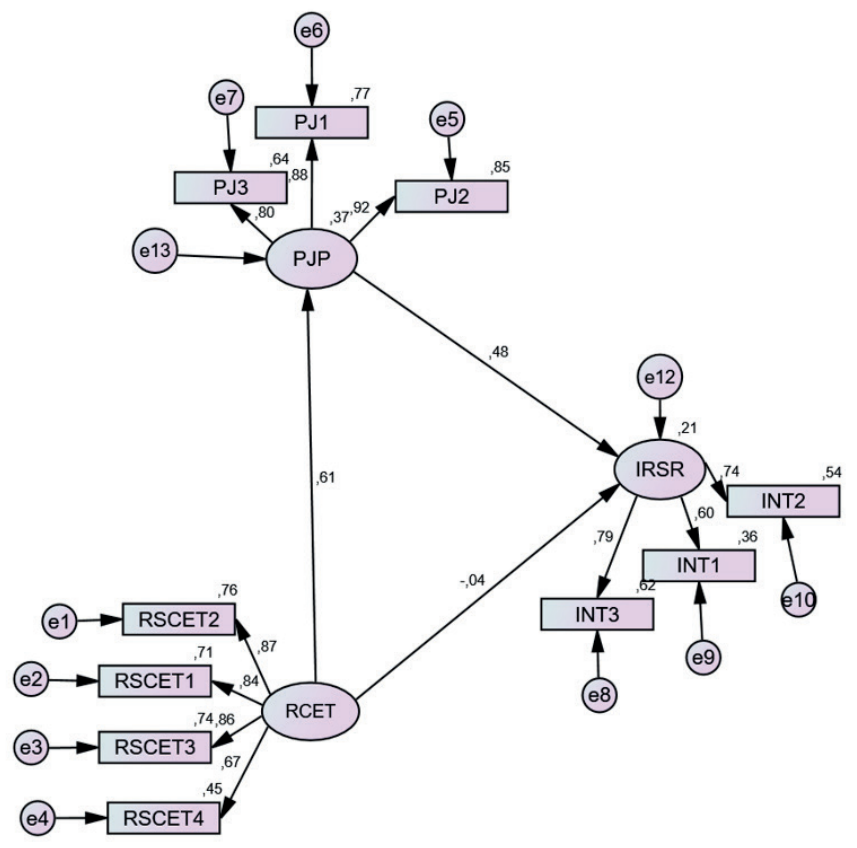

Figura 3. Modelo estructural Fuente: AMOS versión 24.

Tabla 6. Betas estandarizados en la mediación

\begin{tabular}{lccc}
\hline Predictor & Resultado & std Beta & std R \\
\hline RCET & PJP & $0,607^{* * *}$ & \\
PJP & IRSR & $0,484^{* * *}$ & 0,368 \\
RCET & IRSR & $-0,036$ & 0,214 \\
\hline
\end{tabular}

$* * * p<0,001$

Fuente: AMOS versión 24. 


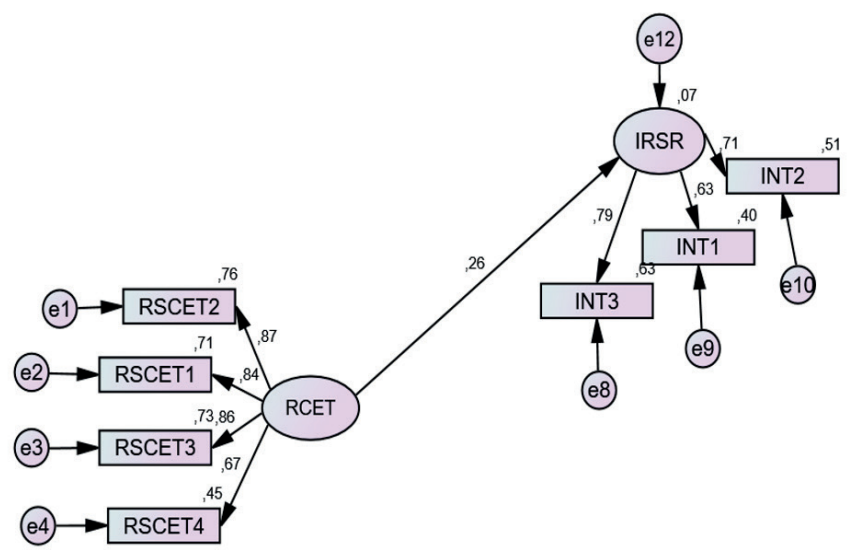

Figura 4. Modelo estructural sin mediación del PJP Fuente: AMOS versión 24.

\section{Conclusiones}

Este estudio analizó los efectos de la RCE en la IRSR, mediados por el PJP. Los resultados confirman la relación positiva entre la RCE y la IRSR. Aunque este efecto de predicción fue significativo, se muestra demasiado débil; esto sugiere que la ética corporativa no es lo suficientemente poderosa como para motivar una intención de compra por sí misma.

De forma alternativa, se estimó un modelo para predecir el IRSR a través de RCE, pero incluyendo el PJP como una variable mediadora. Los resultados indican que a mayor RCE asociada a una empresa mayor será la percepción de justicia respecto a sus precios. Esta relación está alineada con los resultados de estudios previos desarrollados por Semuel y Chandra (2014) y Matute-Vallejo et al. (2011). En consecuencia, se identificó una relación significativa y positiva entre el PJP y la IRSR, como lo respalda la vasta literatura relacionada con el PJP.

Konuk (2019) ha evidenciado la relación positiva entre el PJP y la intención de compra. Sin embargo, al incluir la variable PJP entre la RCE y la relación IRSR, el poder predictivo aumenta considerablemente, lo que evidencia que el efecto RCE se complementa con percepciones de justicia atribuidas al precio, como uno de los principales determinantes del consumo.

Los resultados del estudio tienen algunas implicaciones de gestión. Una vez que se identificó la RCE como un factor que favorece la percepción de justicia en los precios - al ser una variable que también aumenta la intención de una recompra socialmente responsable-, las empresas con RCE deben centrar sus esfuerzos en aumentar el valor percibido de los productos a través de la percepción de justicia en sus precios.

Para aumentar las percepciones de valor de los productos, las empresas deben comunicar sus propiedades no monetarias: los salarios justos, el respeto a los derechos de los trabajadores y consumidores, el cumplimiento de la ley, entre otras. De esta manera se eleva la percepción de justicia en el precio y, por lo tanto, la promoción del consumo responsable, especialmente en las personas que tienen mayores restricciones de inversión en sus compras.

Toda investigación tiene limitaciones para ser discutidas, y este documento no es la excepción. Algunas de las limitaciones que, en opinión del autor, se atribuyen al presente trabajo, así como futuras líneas de investigación derivadas de los resultados se analizan a continuación.

Una de las principales limitaciones está relacionada con el alcance de los resultados, pues los datos se obtuvieron mediante un muestreo de conveniencia, lo que impide su generalización. Otra limitación relacionada con la muestra se refiere al perfil millennial de los encuestados; en su mayoría mujeres jóvenes (67\%) entre 19 y 38 años, sin tener en cuenta las opiniones de otros segmentos de edad y subrepresentando los criterios de los hombres. Por lo tanto, al replicar este trabajo utilizando muestras estratificadas que incluyan la opinión de muestras balanceadas de acuerdo con el perfil de los encuestados, se permitirá la generalización de los resultados.

Los resultados de esta investigación han demostrado que la RCE predice el consumo socialmente responsable, incluyendo la disposición a pagar un poco más por adquirir productos de este tipo de empresas. Asimismo, se ha identificado que este efecto mejora cuando la relación entre la RCE y la intención de recompra está mediada por el PJP. Este hecho establece la posibilidad de modificar esa relación mediante la inclusión de otras variables mediadoras que, como el PJP, apelan a los juicios de justicia entre los consumidores. De hecho, investigaciones anteriores han confirmado que, por ejemplo, la relación entre la RSC y la intención de compra está mediada por el precio justo y la confianza (Semuel y Chandra, 2014). Sin embargo, la presente investigación solo analizó el efecto mediador de la equidad de precios percibida; por lo tanto, se sugiere que la investigación futura explore este efecto mediador en combinación con otras variables mediadoras.

Dado que se confirmaron los efectos del binomio estratégico estudiado, los resultados sugieren que si los consumidores no perciben la justicia a través de las acciones éticas de las empresas, los efectos sobre el precio serían opuestos a los encontrados en este análisis. Así, se espera que las futuras líneas de investigación incluyan variables antagónicas que afecten negativamente a la RCE y al PJP. Atendiendo entonces el comportamiento de 
compra del consumidor, ¿qué pasaría si acciones corporativas no éticas fueran expuestas?, ¿cómo percibirían entonces los consumidores un aumento de precios?

\section{Conflicto de intereses}

Los autores declaran no tener ningún conflicto de intereses.

\section{Bibliografía}

Abrantes, D., Gonçalves, M. y Dias, M. (2010). Corporate social responsibility and consumers' perception of price. Social Responsibility Journal, 6(2), 208-221. https://doi.org/10.1108/17471111011051720

Amezcua, B., Briseño, A., Ríos, T. y Ayala, E. (2018). La disposición a pagar más por productos vinculados a la RSE: evidencia de un análisis conjunto en México. Contaduría y Administración, 63(2), 1-21. https://doi.org/10.22201/fca.24488410e.2018.1013

Arellano-Gault, D. (2019). Government corruption: An exogenous factor in companies' victimization? Public Integrity, 21(2), 141-160. https://doi.org/10.1080/10999922.2018.1433425

Arli, D. I. y Tjiptono, F. (2018). Consumer ethics, religiosity, and consumer social responsibility: Are they related? Social Responsibility Journal, 14(2), 302-320. https://doi.org/10.1108/SRJ-03-2016-0036

Baumgartner, H. y Homburg, C. (1996). Applications of structural equation modeling in marketing and consumer research: $A$ review. International Journal of Research in Marketing, 13(2), 139161. https://doi.org/10.1016/0167-8116(95)00038-0

Berens, G., Van Riel, C. B. M. y Van Bruggen, G. H. (2005). Corporate associations and consumer product responses: The moderating role of corporate brand dominance. Journal of Marketing, 69(3), 35-48. https://doi.org/10.1509/jmkg.69.3.35.66357

Berry, L. L., Seiders, K. y Grewal, D. (2002). Understanding service convenience. Journal of Marketing, 66(3), 1-17. https://doi.org/10.1509/jmkg.66.3.1.18505

Bolton, L. E., Warlop, L. y Alba, J. W. (2003). Consumer perceptions of price (un)fairness. Journal of Consumer Research, 29(4), 474-491. https://doi.org/10.1086/346244

Brown, T. J. y Dacin, P. A. (1997). The company and the Product: Corporate associations and consumer product responses. Journal of Marketing, 61(1), 68-84. https://doi.org/http://dx.doi.org/10.2307/1252190

Carroll. (1979). A three-dimensional conceptual model of corporate performance. Academy of Management Review, 4(4), 497-505. https://doi.org/10.5465/AMR.1979.4498296

Carvalho, S. W., Sen, S., de Oliveira Mota, M. y de Lima, R. C. (2010). Consumer reactions to CSR: A Brazilian perspective. Journal of Business Ethics, 91(S2), 291-310. https://doi.org/10.1007/s10551-010-0620-0

Chark, R. (2019). Price fairness in the era of the sharing economy. Cornell Hospitality Quarterly, 60(3), 200-211. https://doi.org/10.1177/1938965518790221

Chin, W. W. (1998). Commentary. Issues and opinion on structural equation modeling. MIS Quarterly, 22(1), 7-16.

Culiberg, B. y Mihelic, K. K. (2016). Three ethical frames of reference: Insights into Millennials' ethical judgements and intentions in the workplace. Business Ethics, 25(1), 94-111. https://doi.org/10.1111/beer.12106

De Los Salmones, M. D. M. G., Crespo, A. H. y Del Bosque, I. R. (2005). Influence of corporate social responsibility on loyalty and valuation of services. Journal of Business Ethics, 61(4), 369-385. https://doi.org/10.1007/s10551-005-5841-2
Díaz, R. E. P., Díaz, L. R. y González, R. I. (2017). Validación de una escala breve de individualismo-colectivismo. Iberoamericana, 25(1), 30-40.

Dopico, P. A., Rodríguez, D. R. y González, V. E. (2014). Valoración de la RSC por el consumidor y medición de su efecto sobre las compras. Revista de Administração de Empresas, 54(1), 39-52. https://doi.org/10.1590/S0034-759020140105

Elgammal, W., El-Kassar, A.-N. y Messarra, L. C. (2018). Corporate ethics, governance and social responsibility in MENA countries. Management Decision, 56(1), 273-291. https://doi.org/10.1108/MD-03-2017-0287

Ferrell, O. C., Harrison, D. E., Ferrell, L. y Hair, J. F. (2019). Business ethics, corporate social responsibility, and brand attitudes: An exploratory study. Journal of Business Research, 95, 491-501. https://doi.org/10.1016/j.jbusres.2018.07.039

Gómez, N. B. y Martínez, D. R. (2016). Los valores éticos en la responsabilidad social corporativa. Anagramas rumbos sentidos comun, 14(28), 33-49.

Grewal, D., Krishnan, R., Baker, J. y Borin, N. (1998). The effect of store name, brand name and price discounts on consumers' evaluations and purchase intentions. Journal of Retailing, 74(3), 331-352. https://doi.org/10.1016/S0022-4359(99)80099-2

Habel, J., Schons, L. M., Alavi, S. y Wieseke, J. (2015). Warm glow or extra charge? The ambivalent effect of corporate social responsibility activities on customers' perceived price fairness. Journal of Marketing, 80(1), 84-105. https://doi.org/10.1509/jm.14.0389

Hair, J., Hult, T., Ringle, C. y Sarstedt, M. (2017). A primer on partial least squares structural equation modeling (PLS-SEM). Thousand Oaks: Sage Publications.

Higuchi, A. (2015). Características de los consumidores de productos orgánicos y expansión de su oferta en Lima. Apuntes, 42(77), 5789.

Jung, S., Cho, H. J. y Jin, B. E. (2020). Does effective cost transparency increase price fairness? An analysis of apparel brand strategies. Journal of Brand Management, 27(5), 495-507. https://doi.org/10.1057/s41262-020-00191-w

Koellner, E. C. (2019). Perspectiva del consumidor millennial de la responsabilidad social empresarial de la industria automotriz en México. Revista Raites, 5(10), 56-75.

Konuk, F. A. (2019). The influence of perceived food quality, price fairness, perceived value and satisfaction on customers' revisit and word-of-mouth intentions towards organic food restaurants. Journal of Retailing and Consumer Services, 50, 103-110. https://doi.org/10.1016/j.jretconser.2019.05.005

Maignan, I. (2001). Consumers' perceptions of corporate social responsibilities: A cross-cultura I comparison. Journal of Business Ethics, 30, 57-72.

Maignan, I. y Ferrell, O. C. (2004). Corporate social responsibility and marketing: An Integrative Framework. Journal of the Academy of Marketing Science, 32(1), 3-19. https://doi.org/10.1177/0092070303258971

Marquina-Feldman, P. y Reficco, E. (2015). Impacto de la responsabilidad social empresarial en el comportamiento de compra y disposición a pagar de consumidores bogotanos. Estudios Gerenciales, 31(137), 373-382. https://doi.org/10.1016/j.estger.2015.10.001

Martínez, P. y Rodríguez del Bosque, I. (2013). CSR and customer loyalty: The roles of trust, customer identification with the company and satisfaction. International Journal of Hospitality Management, 35, 89-99. https://doi.org/10.1016/j.ijhm.2013.05.009

Matute-Vallejo, J., Bravo, R. y Pina, J. M. (2011). The influence of corporate social responsibility and price fairness on customer behaviour: Evidence from the financial sector. Corporate Social Responsibility and Environmental Management, 18(6), 317-331. https://doi.org/10.1002/csr.247 
Peloza, J., White, K. y Shang, J. (2013). Good and guilt-free: The role of self-accountability in influencing preferences for products with ethical attributes. Journal of Marketing, 77(1), 104-119. https://doi.org/10.1509/jm.11.0454

Semuel, H. y Chandra, S. S. (2014). The analysis of corporate social responsibility implementation effects towards price fairness, trust and purchase intention at oriflame cosmetics product in Surabaya. Procedia - Social and Behavioral Sciences, 155, 42-47. https://doi.org/10.1016/j.sbspro.2014.10.253

Sen, S. y Bhattacharya, C. B. (2001). Does doing good always lead to doing better? Consumer reactions to corporate social responsibility. Journal of Marketing Research, 38(2), 225-243. https://doi.org/10.1509/jmkr.38.2.225.18838

The World Bank (2020). World Bank Country and Lending Groups . Recuperado el 21 de enero de 2020, de: https://n9.cl/ugb9
Tian, Z., Wang, R. y Yang, W. (2011). Consumer responses to corporate social responsibility (CSR) in China. Journal of Business Ethics, 101(2), 197-212. https://doi.org/10.1007/s10551-010-0716-6

Xia, L., Monroe, K. B. y Cox, J. L. (2004). The price is unfair! A conceptual framework of price fairness perceptions. Journal of Marketing, 68(4), 1-15. https://doi.org/10.1509/jmkg.68.4.1.42733

Zeithaml, V. A. (1988). Consumer perceptions of price, quality, and value: A means-end model and synthesis of evidence. Journal of Marketing, 52(3), 2-22. https://doi.org/10.2307/1251446

Zietsman, M. L., Mostert, P. y Svensson, G. (2019). Perceived price and service quality as mediators between price fairness and perceived value in business banking relationships: A microenterprise perspective. International Journal of Bank Marketing, 37(1), 2-19. https://doi.org/10.1108/IJBM-07-2017-0144 